\title{
Uso de laboratorios virtuales como estrategia didáctica para el aprendizaje activo
}

\author{
Larisa Elizabeth Lara Ramírez \\ larisa.lara@academicos.udg.mx \\ https://orcid.org/0000-0002-5564-6808 \\ María Isabel Pérez Vega \\ misabel.perez@academicos.udg.mx \\ https://orcid.org/0000-0002-0708-5022 \\ Paola Trinidad Villalobos Gutiérrez \\ paola.villalobos2452@academicos.udg.mx \\ https://orcid.org/0000-0001-5689-3619 \\ Virginia Villa-Cruz \\ virginia.villa@academicos.udg.mx \\ https://orcid.org/0000-0002-1442-8548 \\ Juan Onofre Orozco López \\ juan.onofre@academicos.udg.mx \\ https://orcid.org/0000-0002-0711-6538 \\ Luis Javier López Reyes \\ luis.lreyes@academicos.udg.mx \\ https://orcid.org/0000-0002-3534-1070 \\ Universidad de Guadalajara / Centro Universitario de los Lagos \\ Lagos de Moreno - México
}

\section{RESUMEN}

Los laboratorios virtuales son herramientas didácticas que fomentan el desarrollo de habilidades blandas y disciplinares, ya que permiten a los estudiantes poner en práctica sus conocimientos teóricos y experimentar cuantas veces lo requieran. En el presente trabajo se analizó la contribución del uso de los laboratorios virtuales, como parte de una estrategia didáctica para el aprendizaje activo en alumnos de Ingeniería Bioquímica. Se diseñaron instrumentos y actividades para medir el nivel de conocimiento o percepción de la tecnología digital y para evaluar el impacto del uso de laboratorios virtuales. Se observó que el 100\% de los alumnos que conformaron el grupo control, conocen lo que es la tecnología digital, mientras que ese porcentaje disminuyó en el grupo experimental a $79 \%$. Aunque el $100 \%$ de los alumnos, de ambos grupos, identifican qué es un 
laboratorio virtual y su importancia como plataforma de ensayo-aprendizaje, se obtuvieron respuestas divididas con respecto a si el uso de laboratorios virtuales les ayudó en su aprendizaje, un $57.1 \%$ mencionó que sí, mientras que el $42.9 \%$ restante mencionó que no. Por lo que se considera que los laboratorios virtuales son una herramienta útil en el proceso enseñanza aprendizaje.

Palabras clave: laboratorios virtuales; aprendizaje activo; TIC; competencias STEM. 


\title{
Use of virtual laboratories as a teaching strategy for active learning
}

\begin{abstract}
Virtual laboratories are educational tools that encourage the development of soft and disciplinary skills, since they allow students to put their theoretical knowledge into practice and experiment as many times as they require. In the present work, the contribution of the use of virtual laboratories was analyzed, as part of a didactic strategy for active learning in students of Biochemical Engineering. Instruments and activities were designed to measure the level of knowledge or perception of digital technology and to evaluate the impact of the use of virtual laboratories. It was observed that $100 \%$ of the students who made up the control group know what digital technology is, while that percentage decreased in the experimental group at $79 \%$. Although $100 \%$ of the students, from both groups, identify what a virtual laboratory is and its importance as a testinglearning platform, divided responses were obtained regarding whether the use of virtual laboratories helped them in their learning, $57.1 \%$ said yes, while the remaining $42.9 \%$ mentioned that they did not. So that virtual laboratories are considered to be a useful tool in the teaching-learning process.
\end{abstract}

Keywords: virtual laboratories; active learning; ICT; STEM skills;

Artículo recibido: 15 enero 2022 Aceptado para publicación: 08 febrero 2022 Correspondencia: virginia.villa@academicos.udg.mx Conflictos de Interés: Ninguna que declarar 


\section{INTRODUCCIÓN}

Los egresados de programas de estudio del área de Ciencia, Tecnología, Ingeniería y Matemáticas (STEM, por sus siglas en inglés) deben mostrar dominio de competencias y habilidades propias de su carrera, que han sido establecidas internacionalmente en el Washington Accord (IEA, 2022) y aceptadas por organismos acreditadores (CEAB, 2022; ABET, 2022; CACEI, 2022). Entre los atributos de egreso de un estudiante de ingeniería están, por un lado, las conocidas como competencias duras: i) aplicar conocimientos y técnicas, ii) diseñar sistemas, componentes o procesos que satisfagan necesidades específicas, iii) realizar pruebas, mediciones y experimentos estándar y de analizar e interpretar los resultados para mejorar los procesos; todo ello para resolver problemas propios de su formación. Por otro lado, están las conocidas como habilidades blandas: i) pensamiento crítico, ii) comunicación efectiva en forma escrita, oral y gráfica, así como identificar y utilizar la literatura técnica adecuada y, iii) funcionar eficazmente como miembro y como líder en equipos técnicos.

En este sentido, las instituciones de educación superior reconocen la importancia de fomentar el desarrollo de estas competencias y habilidades en sus estudiantes. En particular, el programa educativo de Ingeniería Bioquímica (IBIO) que se ofrece en el Centro Universitario de los Lagos (CULagos) de la Universidad de Guadalajara (UdeG), incluye estos criterios y parámetros, como parte de su plan de estudios para lograr el objetivo de brindar una formación integral a los estudiantes. Así, mediante la implementación de algunas propuestas didácticas y diversas metodologías activas se puede contribuir a mejorar el aprendizaje de los estudiantes de un tema específico y en el desarrollo de ciertas competencias y habilidades.

El programa educativo de IBIO combina la parte teórica y práctica para el aprendizaje en los alumnos, siendo la práctica parte fundamental en el desempeño del estudiante, donde es indispensable el uso de laboratorios. El programa toca muchos tópicos de enseñanza desde la física, la química, la biología, hasta la microbiología, por lo que se requieren diversos tipos de laboratorios. Actualmente el uso de laboratorios virtuales (LV) representa una oportunidad de aprendizaje activo para los estudiantes, ya que les permite contextualizar un tema o proyecto a desarrollar. Se puede mencionar que funcionan como una plataforma de ensayo durante el aprendizaje del estudiante y con esto se evitan los 
posibles riesgos o accidentes que surgen al usar un laboratorio presencial sin previo estudio del tema a desarrollar.

En este sentido, implementar propuestas didácticas, con metodologías activas, son alternativas que contribuyen a incrementar el aprendizaje en los estudiantes, logrando que desarrollen tanto habilidades o competencias duras y blandas. Actualmente, los LV forman parte de las estrategias didácticas ya que se estimula la enseñanza virtual. Así, los LV se pueden utilizar como complemento o sustituto del laboratorio convencional para mejorar el conocimiento de los estudiantes (Chan \& Fok, 2009; Kapilan, Vidhya and Gao, 2021). Los LV fomentan el autoaprendizaje, responsabilidad e independencia en el estudiante, ya que se pueden utilizar de manera flexible; también contribuyen a prevenir riesgos de accidentes en el laboratorio presencial, porque de alguna forma el estudiante ha ensayado virtualmente la práctica; pueden ser económicos comparados a los laboratorios convencionales, por los costos operativos del propio laboratorio convencional.

Un estudio realizado en Dinamarca muestra que los LV permiten el aprendizaje interactivo, para la comprensión del funcionamiento de los sistemas y equipos biológicos y bioquímicos utilizados en los laboratorios; así como, para evaluar y analizar los resultados obtenidos de los experimentos realizados de manera virtual. Se busca que los estudiantes desarrollen una mayor confianza y habilidades al utilizarlos, se sientan cómodos con el manejo del instrumental y equipo de laboratorio y participen activamente en el desarrollo de experimentos mediante el uso de los LV, de manera que con estas actividades mejoren su desempeño en los laboratorios convencionales (Dyrberg et al., 2016; Kapilan, Vidhya and Gao, 2021).

El programa de IBIO es uno de los ocho programas de tipo STEM ofertado desde hace 20 años por el CULagos, ubicado en la región Altos Norte del estado de Jalisco. El CULagos es una dependencia regional que forma parte de la red universitaria de la UdeG, conformada por 15 centros universitarios (6 temáticos y 9 regionales). La UdeG es considerada la institución de educación superior (IES) más importante en el occidente de la República Mexicana, y la segunda, de México. En el CULagos como en la mayoría de las dependencias de IES públicas, se cuenta con infraestructura limitada para enfrentar el reto de cubrir de manera satisfactoria todas las actividades planteadas dentro del plan de estudios de un programa educativo tipo STEM. Los espacios destinados para laboratorio 
son pequeños y escasos, además, la cantidad de equipo didáctico, materiales y/o reactivos, es limitada. Por lo tanto, el trabajo aquí presentado tiene sentido como estudio piloto, por el potencial que representa para fomentar el aprendizaje activo en los estudiantes.

La propuesta didáctica se implementó utilizando metodologías activas en un contexto docente durante el ciclo escolar 2021B con estudiantes del segundo ciclo de IBIO. La intervención se llevó a cabo en 4 grupos que cursaron materias del área de formación básica: i) Tecnología de la Información y Comunicación, ii) Biología Celular. Se designó un grupo control y un grupo experimental en cada materia. Para realizar el presente estudio se establecieron los siguientes objetivos:

\section{Objetivo general}

- Analizar la contribución del uso de los LV en el desarrollo de las competencias transversales y disciplinares del ingeniero bioquímico.

\section{Objetivos específicos}

- Evaluar el nivel de conocimiento y percepción de los estudiantes sobre el uso de LV como una tecnología digital que favorece el aprendizaje activo.

- Evaluar el uso de LV como metodología activa en el aprendizaje significativo de la estructura de las células procariotas y eucariotas.

\section{ESTRATEGIAS METODOLÓGICAS O MATERIALES Y MÉTODOS}

En la propuesta didáctica se consideró, por un lado, analizar el desarrollo de competencias transversales (blandas), para lo cual se diseñaron instrumentos y actividades que evalúan el nivel de conocimiento y percepción de los estudiantes sobre el uso de LV. Por otro lado, analizar el desarrollo de competencias disciplinares (duras), mediante formularios y actividades que evalúan el efecto del uso de LV como metodología activa en el aprendizaje significativo de la estructura de las células procariotas y eucariotas. La implementación de este estudio consistió de dos etapas, que se describen a continuación: En la primera etapa, se trabajó con dos grupos que cursaron la materia de Tecnología de la Información y Comunicación, un grupo control y un grupo experimental, integrados por 17 y 19 estudiantes respectivamente. Se realizaron diferentes actividades para fomentar el desarrollo de competencias transversales como trabajo en equipo, uso de tecnologías digitales, y comunicación efectiva. En esta etapa se aplicaron pre-test y posttest como instrumentos de observación. El pre-test fue aplicado como diagnóstico en ambos grupos para evaluar el nivel de conocimiento de los estudiantes sobre el uso de 
tecnologías digitales y LV como apoyo para potencializar su aprendizaje. Las actividades consistieron en lectura de documentos, revisión de videos (h5p o edpuzzle), elaboración de mapas mentales, participación en debates y elaboración de un video en equipo para presentar las ventajas del uso de los LV, todas las actividades fueron evaluadas mediante el uso de rúbricas. El post-test fue aplicado para evaluar el conocimiento de los alumnos sobre los LV. Los cuestionarios fueron basados en el contenido del módulo II: Tecnologías para la Información y Comunicación (TIC), en el subtema Nuevas Tecnologías Digitales, y más específicamente en el tema Laboratorios Virtuales.

En la segunda etapa, se trabajó con 2 grupos que cursaron la materia de Biología Celular, un grupo control y un grupo experimental, integrados por 22 y 21 estudiantes respectivamente. Se realizaron diferentes actividades, en ambos grupos, como lectura de artículos, revisión videos, consulta de información en diferentes fuentes bibliográficas, para contribuir en el desarrollo de una competencia disciplinar: conocer y comprender la estructura de las células procariotas y eucariotas; por otro lado, al grupo experimental se le proporcionó enlaces de acceso a LV disponibles en la web de la Universidad Nacional Autónoma de México (UNAM, 2022) y en plataformas educativas (CUVSI, 2022), para desarrollar habilidades en el uso y manejo del microscopio e identificación de las estructuras celulares. Posteriormente se aplicó un examen de conocimiento a ambos grupos, para evaluar el conocimiento y comprensión sobre el tema. Al grupo experimental adicionalmente se les aplicó una encuesta para valorar su percepción sobre el uso de LV en el proceso enseñanza aprendizaje.

Por último, los resultados obtenidos de ambas etapas fueron analizados de manera cualitativa y cuantitativa.

\section{RESULTADOS Y DISCUSIÓN}

A continuación se presentan los resultados obtenidos de la aplicación del pre-test a los grupos control y experimental que cursaron la materia de "Tecnología de la Información y Comunicación", respecto al conocimiento y uso de los LV. En la figura 1 se muestra el nivel de conocimiento de los estudiantes sobre el uso de tecnologías digitales, el 100\% del grupo control identifica que es una tecnología digital mientras que el $21 \%$ del grupo experimental no es capaz de discernir que es una tecnología digital. 

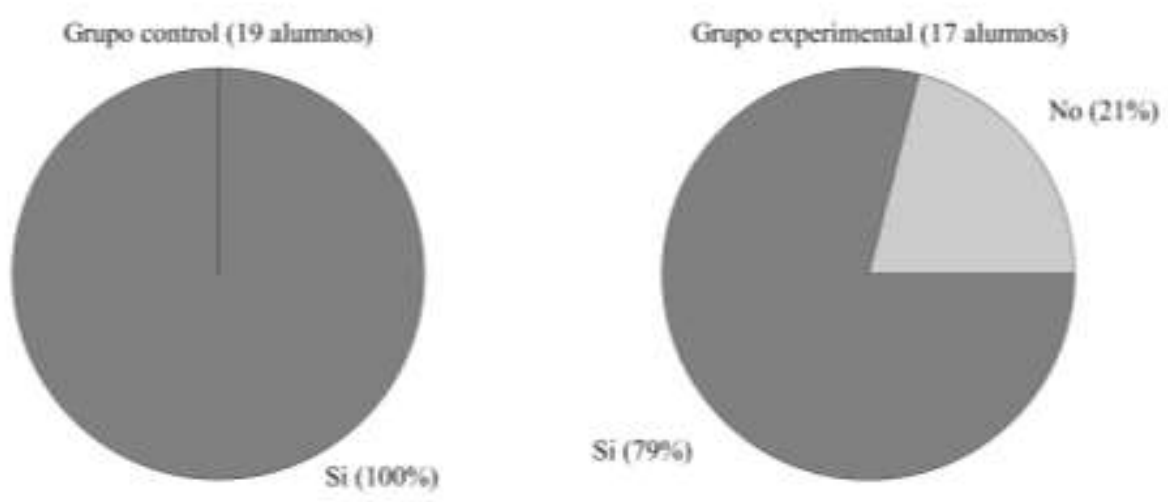

Figura 1. Respuestas de los grupos a la pregunta ¿Sabes qué es una tecnologia digital?

Este pre-test también mostró que la mayoría de los alumnos de ambos grupos relacionan primordialmente la tecnología digital con aplicaciones o métodos para procesar información, seguido posteriormente de la relación con dispositivos electrónicos (teléfonos móviles y tabletas); en tercer nivel lo relacionan con medios de comunicación digital: correo electrónico y redes sociales como se puede visualizar en la figura 2.

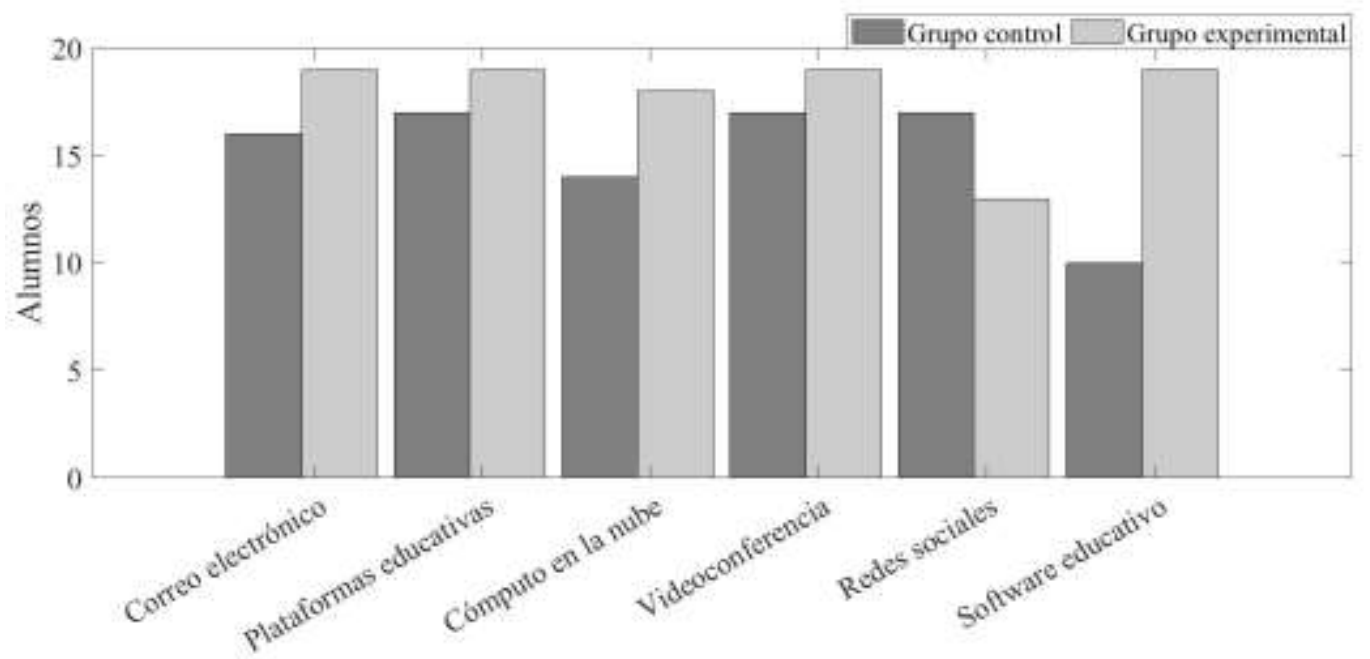

Figura 2. Respuestas de los grupos a la pregunta ¿Cuáles herramientas digitales han utilizado?

Por otro lado en el pre-test se identificó que el 100\% de los alumnos de ambos grupos (control y experimental) saben lo que es un LV y lo relacionan con el uso de software para realizar prácticas de laboratorio sustituyendo la realización física de la misma reduciendo los riesgos de accidente y principalmente utilizados previo a la realización de prácticas reales. La razón es porque la totalidad de los alumnos de ambos grupos utilizaron los LV para realizar prácticas relacionadas con la asignatura de química 
(materia cursada previamente a tecnología de la información y comunicación, con base en su plan de estudios).

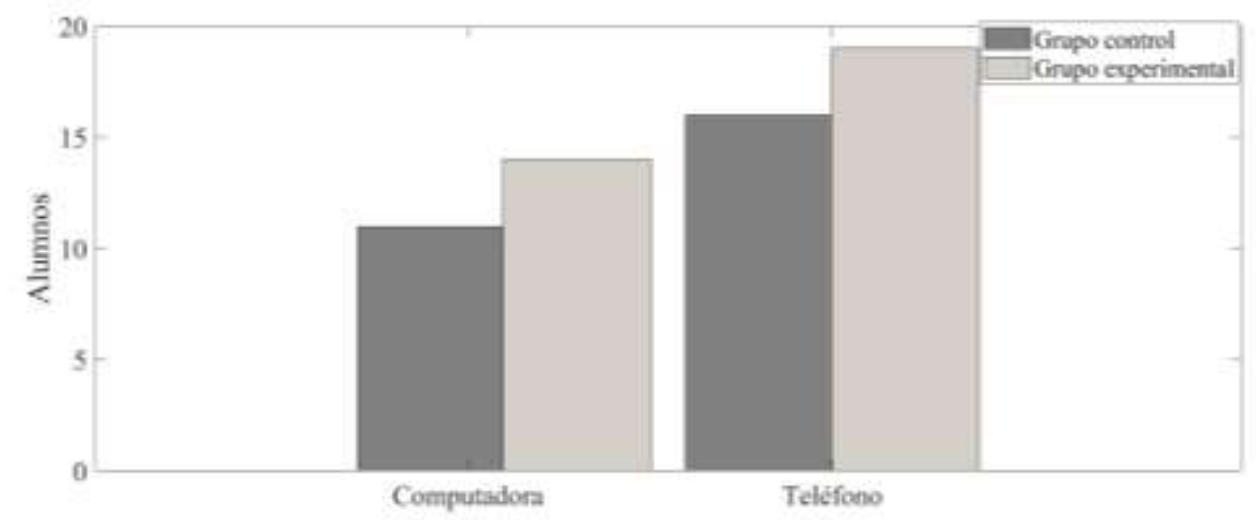

Figura 3. Respuestas de los grupos a la pregunta ¿Qué tecnologia digital utilizas con mayor regularidad?

Se pudo identificar en los resultados obtenidos del pre-test que la tecnología digital más utilizada actualmente es el teléfono celular como se muestra en la figura 3; lo cual puede atribuirse a la facilidad de acceso y transporte de dicho dispositivo.

Algunas preguntas de tipo abierto en el pre-test permiten a los alumnos enlistar las ventajas que ellos identifican respecto a la utilización de LV, ambos grupos coinciden en las siguientes: es posible realizar la práctica sin tener que interactuar de manera presencial, disponibilidad de tiempo para realizar la actividad asignada, facilidad para repetir las prácticas, no hay riesgo de accidentes. Por su parte, entre las limitaciones que enlistan se encuentran las siguientes: no hay interacción con compañeros o profesor y cuando hay dudas no tienen a quien preguntar y por tanto la comprensión del proceso no es la mejor, no aprenden el manejo práctico de los instrumentos y materiales, se requiere de equipo de cómputo y conexión a internet.

De acuerdo a los resultados obtenidos en la encuesta aplicada a los alumnos del curso de Biología Celular del ciclo escolar 2021-B (grupo experimental), sobre su percepción del uso de LV, se obtuvo que el 73\% de los alumnos había usado previamente un laboratorio virtual, para el $82 \%$ de los alumnos los materiales digitales proporcionados fueron comprensibles y adecuados, al 59\% de los estudiantes los LV les ayudaron en su proceso de enseñanza-aprendizaje y para el $73 \%$ las tecnologías digitales les ayudó a la comprensión del tema, como se muestra en la figura 4. 


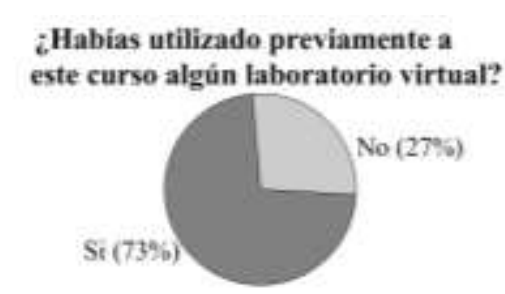

El uso de los laboratorios virtuales en el procese ensef̂anza-aprendizaje te ayudó:

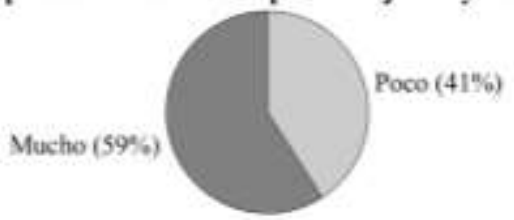

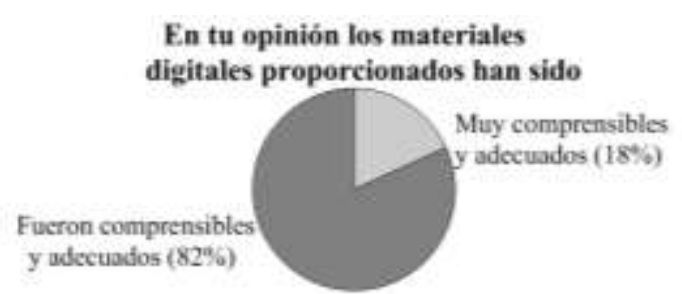

El uso de las tecnologias digitales proporcionadas te ayudó a la mejor comprensión del tema:

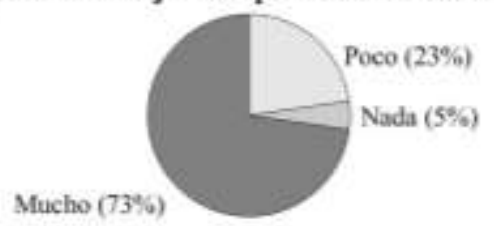

Figura 4. Resultados de la encuesta aplicada al grupo experimental, en el que se implementó el uso de LV

Por otro lado, se muestran las respuestas más relevantes que dieron los alumnos con respecto a las ventajas de utilizar un $L V$, i) se puede tener acceso a la información de cualquier cosa a tu alcance desde cualquier lugar, la información que brindan es buena, comprensible y clara, ii) se optimiza el tiempo y los materiales al realizar las prácticas de manera virtual, iii) son dinámicos y muy visuales, lo cual permite una mayor comprensión de los temas previamente vistos en clase y, por otro lado, posibilita revisar los procedimientos y observar con detalle diversos tipos celulares cuantas veces se requiera $\mathrm{y}$, algunos programas presentan las partes de las células, sus nombres y funciones de manera que ayuda a la mejor identificación de las estructuras celulares, iv) son seguros ya que elimina por completo la posibilidad de un accidente que resulte peligroso para quien experimenta, v) permiten aprender y familiarizarse con los materiales, procedimientos y estructuras celulares, vi) ayuda a que no exponerse a contagios por covid.

Con respecto al uso de los LV como apoyo en los cursos, las respuestas más relevantes fueron las siguientes: i) a través de la interacción con los LV es posible expandir nuestro conocimiento de los temas tratados en clase y mientras se siga con la modalidad en línea, resultan de gran ayuda para la comprensión y visualización de los procesos que se pueden desarrollar en los laboratorios de manera dinámica, ii) contribuyen a un mejor desempeño y rendimiento en nuestra formación, iii) representan una buena manera de tener otro punto de vista, porque dan un acercamiento a los laboratorios y prácticas cuando las circunstancias no lo permiten. iv) representan una opción relativamente fácil de 
desarrollar procesos y observar algo que hasta el momento solo se podía visualizar en un laboratorio físico.

Entre las principales dificultades para tener acceso a los LV expresaron lo siguiente: i) algunas páginas tardan mucho en cargar y en algunos casos al final marcaba error, ii) los programas son muy pesados lo que hace que sus equipos se vuelvan más lentos y no permiten su descarga en la computadora, iii) no contar con equipo de cómputo propio iv) dificultad en el acceso a internet en las localidades que residen, lo que implica un gasto adicional a las finanzas del hogar.

En la figura 5 se muestra el resultado de la aplicación del examen de conocimiento, en ambos grupos, que evaluó el conocimiento y comprensión sobre la identificación de las estructuras células procariotas y eucariotas. La intención del examen fue medir cómo los LV influyen de forma significativa en el aprendizaje de un tema disciplinar que contiene la asignatura de Biología celular. El gráfico muestra la comparativa del grado de conocimiento del estudio de las células procariotas y eucariotas en los dos grupos analizados. En el diagrama de caja se puede observar de manera cualitativa que ambos grupos presentan la misma mediana, sin embargo, se aprecia que la distribución de los resultados en el grupo experimental es más compacta comparada con la distribución de resultados del grupo control en el que es más dispersa; esto puede ser atribuido a una combinación de dos factores considerados dentro de esta propuesta didáctica (de aprendizaje activo); por un lado, la secuencia de actividades de aprendizaje realizadas por los estudiantes y por otro lado, el uso de los LV como una herramienta digital para fomentar un aprendizaje significativo de la estructura de las células procariotas y eucariotas en el grupo experimental.
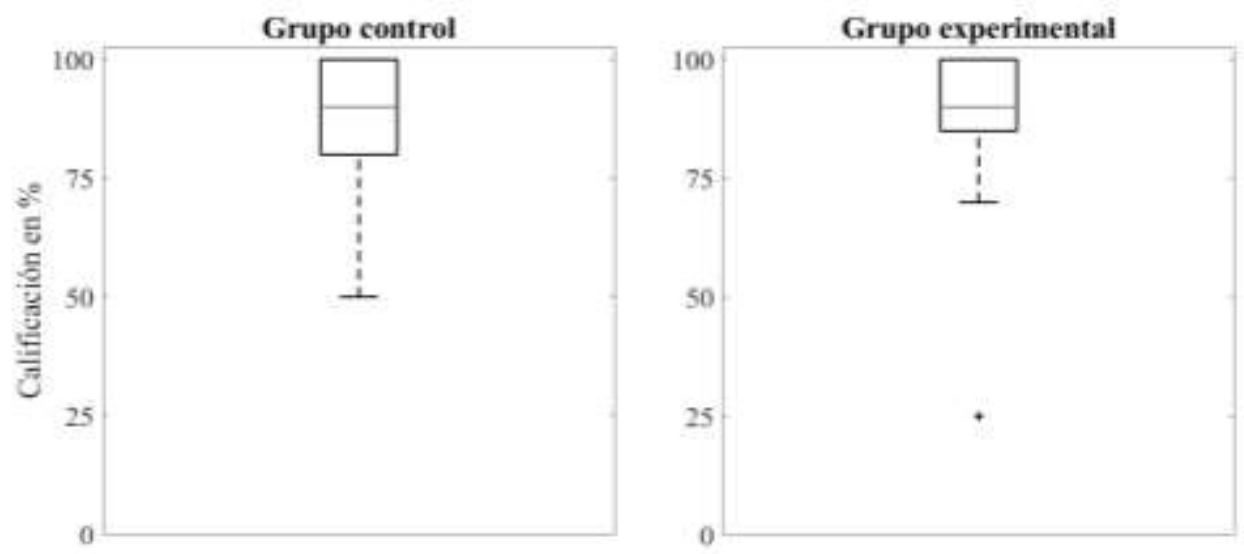

Figura 5. Comparación de resultados obtenidos en la evaluación de conocimientos en los grupos analizados. 
Por último, debe mencionarse que mediante un análisis cuantitativo con estadísticos descriptivos se observa que los resultados del examen de conocimientos son prácticamente idénticos en ambos grupos, con una media y desviación estándar de 87.27 con 15.56 en el grupo control y de 88.09 con 16.91 en el grupo experimental. No obstante, la implementación de esta propuesta didáctica cumplió su propósito como estudio piloto cuyos resultados e información obtenida serán muy valiosos y considerados en futuros estudios docentes.

\section{CONCLUSIÓN O CONSIDERACIONES FINALES}

Estamos en un siglo donde el avance tecnológico y el uso de las TIC's es considerado de dominio público, la respuesta de nuestros alumnos del conocimiento pleno de las tecnologías digitales, nos sitúa dentro de este panorama. Dentro de esas tecnologías digitales los laboratorios virtuales juegan un papel clave en didácticas de enseñanzas activas, por lo que es satisfactorio la respuesta del conocimiento de los LV por el $100 \%$ de nuestros alumnos estudiados.

En asignaturas donde la enseñanza-aprendizaje requiere del uso de laboratorios, se pueden presentar muchos beneficios al usar los LV, como, por ejemplo: los estudiantes, pueden mejorar su comprensión y rendimiento mediante el aprendizaje activo en el campo de la ingeniería. Por su parte, los profesores pueden cumplir los objetivos de aprendizaje propuestos, caso contrario cuando se enfrentan a la falta de material y/o reactivos o en su defecto a la falta de laboratorios presenciales. Por último, la Universidad, se puede beneficiar en la optimización de recursos económicos, disminuyendo el uso y/o instalación de laboratorios presenciales.

El uso de laboratorios virtuales representa un medio de apoyo útil como complemento para la mejor comprensión de los temas vistos en clase. Sin embargo, hay que saber elegirlos y tener en consideración que no pueden sustituir las habilidades prácticas de manipulación de materiales e instrumentos que brinda la experiencia de practicar en un laboratorio real. Por otro lado, el conocer qué son los LV no siempre conlleva al uso de los mismos y más aún, al manejo y aprendizaje en ellos; tal como se observó en los resultados, donde se aprecian opiniones divididas con respecto a si el uso de los LV fue de apoyo en su aprendizaje. Estas diferencias de opiniones se pueden deber a varios factores: como bajo conocimiento cognitivo del alumno, falta de asesoramiento por parte del profesor y limitaciones en conexión e infraestructura, entre otras. Por lo que, es 
importante considerar que estos factores siempre van a existir y que es un compromiso constante, por parte de las entidades educativas actuar sobre ellos y tenerlos en consideración.

\section{LISTA DE REFERENCIAS}

CEAB Accreditation Criteria and Procedures Available online: https://engineerscanada.ca/accreditation/accreditation-resources (consultado el 13 de enero de 2022).

ABET Criteria for Accredition Engineering Program Available online: https://www.abet.org/accreditation (consultado el 13 de enero de 2022).

CACEI Marco de Referencia 2018 Available online: http://www.cacei.org.mx/nvfs/nvfs02/nvfs0210.php (consultado el 13 de enero de 2022).

CHAN, C., \& FOK, W. (2009). Evaluating learning experiences in virtual laboratory training through student perceptions: a case study in Electrical and Electronic Engineering at the University of Hong Kong. engineering education, 4(2), 70-75.

CUVSI 2014 Available online: https://www.cuvsi.com/2014/11/laboratorios-virtualesde-biologia.html\#google_vignette (consultado el 7 de septiembre de 2021)

CUVSI 2016 Available online: https://www.cuvsi.com/2016/02/identificacionmicroscopica-de.html (consultado el 7 de septiembre de 2021).

DYRBERG, N. R., TREUSCH, A. H., \& WIEGAND, C. (2017). Virtual laboratories in science education: students' motivation and experiences in two tertiary biology courses. Journal of Biological Education, 51(4), 358-374.

IEA Washington Accord Available online: https://www.ieagreements.org/accords/washington/ (consultado el 13 de enero de 2022).

KAPILAN, N., VIDHYA, P., \& GAO, X. Z. (2021). Virtual laboratory: A boon to the mechanical engineering education during covid-19 pandemic. Higher Education for the Future, 8(1), 31-46.

UNAM

Available

online:

http://www.objetos.unam.mx/biologia/celulaProcariota/index.html (consultado el 7 de septiembre de 2021). 